\title{
Altered HLA Class I and HLA-G Expression Is Associated with IL-10 Expression in Patients with Cervical Cancer
}

\author{
Josefa Antonia Rodríguez ${ }^{\mathrm{a}}$ Liliana Galeano $^{\mathrm{a}}$ Diana María Palacios ${ }^{\mathrm{b}, \mathrm{c}}$ \\ Constanza Gómez $^{\text {d }}$ Martha Lucía Serrano ${ }^{a}$ María Mercedes Bravo ${ }^{a}$ \\ Alba Lucía Combita ${ }^{\text {a, }}$ \\ ${ }^{a}$ Grupo de Investigación en Biología del Cáncer, Instituto Nacional de Cancerología, b Departamento de Patología, \\ Fundación Santa Fe de Bogotá, 'FFacultad de Medicina, Universidad Nacional de Colombia, y dGrupo de Patología, \\ Instituto Nacional de Cancerología, Bogotá, Colombia
}

\section{Key Words}

Histocompatibility antigen class I - HLA-G • Interleukin-10 •

Cervical cancer

\begin{abstract}
Although high-risk human papillomaviruses (HPVs) are an important risk factor in the etiopathogenesis of cervical cancer, increasing evidence suggests that the ability to avoid immune surveillance seems to be linked to the transforming potential of HPV and a rapid progression to cancer. In other cancer models, IL-10 contributes to impair anti-tumor immune response either by downregulating human leukocyte antigen Class I (HLA-I) expression or by increasing HLA-G expression. To comprehend how these alterations could contribute to evasion of immune surveillance in cervical cancer, we analyzed HLA-I, HLA-G and IL-10 expressions by immunohistochemistry in 63 biopsies from patients with cervical intraepithelial neoplasia III (CIN-III) and cervical cancer. Immunohistochemistry showed absent or weak HLA-I expression in 50/59 cases. In these cases, a high percentage had loss of heterozygosis. IL-10 and HLA-G expression were observed in 46.6 and $27.6 \%$ of cases, respectively. Concurrent upregula-
\end{abstract}

tion of IL-10 was found in $87.5 \%$ of HLA-G positive cases $(p=$ 0.000). Similarly, a significant association between IL-10 expression and HLA-I downregulation was found ( $p=0.028$ ). Finally, we observed higher HLA-G expression in patients with HLA-I downregulation than in those with normal HLA-I expression ( $p=0.004$ ). Our results suggest that, in cervical cancer, the IL-10 expression may induce an immunosuppressive environment by upregulating HLA-G expression and downregulating HLA class I expression.

Copyright $\odot 2011$ S. Karger AG, Basel

\section{Introduction}

Although infection with high-risk human papillomavirus (HPV) is an important risk factor in cervical cancer etiopathogenesis, there is increasing evidence supporting that the immune system plays a pivotal role in determining the outcome of HPV infections [1]. Evidence suggests that immunosuppressed individuals are more susceptible to developing persistent HPV infections and cervical intraepithelial HPV-associated neoplastic lesions [2-4]. This suggests that the ability to avoid immune surveil-

\section{KARGER}

Fax +4161306 1234 E-Mail karger@karger.ch www.karger.com

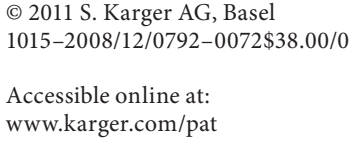

Alba Lucía Cómbita Rojas

Av. 1 No. 9-85

Bogotá (Colombia)

Tel. +57 13340959

E-Mail acombita@ cancer.gov.co 
lance might be linked to the transforming potential of HPV and to the rapid progression of cervical cancer [1].

There are several mechanisms that could explain the complex cancer-host immune interactions and immune escape of cancer cells into the surrounding environment. One mechanism frequently linked to tumor escape from host immune surveillance is an alteration of human leukocyte antigen (HLA) expression on tumor cells [5, 6]. Thus, a complete loss of human HLA class I molecules results in resistance to cytotoxic T lymphocyte (CTL)-mediated lysis, but it in turn renders tumor cells susceptible to natural killer (NK) cell-mediated killing. Total or partial loss of HLA class I molecules expression has been reported in different human tumors [7-9]. In cervical cancer, loss of HLA class I occurs frequently and is mainly caused by genetic aberrations in the $6 \mathrm{p} 21.3$ region [10-13]. It has been reported that at least $50 \%$ of multiple HLA allele loss is caused by loss of heterozygosis (LOH) [14, 15].

Among other strategies that have been developed by tumor cells to avoid recognition by different immune effectors, the non-classical HLA-G seems to be one of the most powerful molecules for the suppression of the innate and/or adaptive immune response by multiple pathways of the immune system [16]. In fact, it has been reported that HLA-G inhibits cytotoxicity of CD8+ T cells and also NK cells, as well as T-cell alloproliferation [17, 18]. Additionally, HLA-G affects dendritic cell maturation, migration, trafficking, antigen presentation and their cross-talk with T and NK cells [19]. Finally, it could also modulate and shift the release of cytokine productions from T-helper Th1 to Th2 profile [20, 21]. Numerous studies have indicated that HLA-G expressed in cancer patients contributes to tumor progression and could be a significant prognostic factor [22].

In cervical cancer it is well-known that progression is associated with a shift from Th1 to Th2 cytokine production. This shift toward a Th2 cytokine profile, characterized by IL-10 secretion, is associated with progression of premalignant lesions to cancer $[23,24]$. It has been reported that IL-10 can contribute to an impaired anti-tumor immune response either by downregulating HLA class I expression allowing tumor escape from lysis mediated by CTLs [25], or by increasing HLA-G expression allowing HLA class I-deficient cells to escape from NKmediated lysis through interaction with killer inhibitory receptors on NK cells [26-29]. In lung cancer, IL-10 secretion can contribute to the progression by downregulating HLA class I expression and selective HLA-G induction on tumor cells [30-32]. In cervical cancer, the HLA-G and IL-10 expression has been shown to be higher in tu- mor cells than in normal cervix, and may be involved in early carcinogenesis. However, there is no clear association between HLA-G and IL-10 expression in cervical cancer [33]. To evaluate the contribution of these mechanisms in cervical cancer, we analyzed the expression of HLA class I, HLA-G and IL-10 at the tumor cell surface in biopsies of patients with CIN III and invasive cervical cancer.

\section{Materials and Methods}

\section{Study Population}

Sixty-three patients with CIN III and invasive cervical cancer stage IBI-IVB according to the International Federation of Gynecologists and Obstetrics (FIGO), attending the outpatient gynecology clinic at the Instituto Nacional de Cancerología (INC), in Bogotá, Colombia, from October 2004 to October 2005, were enrolled in this study. Patients were not included if they had undergone any treatment before, if they showed prior or concurrent second malignancies or if they had been pregnant. All individuals were subjected to a short interview concerning medical, gynecologic and sexual history. Written informed consent was obtained from all patients according to the guidelines of the institutional review board. This study was reviewed and approved by the INC Medical Ethics Committee.

\section{Tissue and Blood Samples}

During the first gynecological examination, cervical scrapes were collected from each patient using a spatula and a brush and placed in tubes containing $5 \mathrm{ml}$ phosphate-buffered saline $(1 \times$ PBS) and $0.05 \%$ thimerosal to determine the presence of HPV DNA. Cervical biopsies were obtained and cryopreserved in Tissue Freezing Medium (Triangle Biomedical Sciences, Durham, N.C., USA) for further LOH analyses and immunostaining procedures. Blood samples were also collected and peripheral blood mononuclear cells (PBMCs) were isolated from fresh heparinized blood by Ficoll-Hypaque density gradient centrifugation (Sigma) and used as normal control for $\mathrm{LOH}$ analysis.

\section{Microdissection and DNA Extraction}

To minimize the possibility of contaminating the tumor samples with normal cells, the following protocol for tumor microdissection was used. Fixed cryopreserved tissue sections of $4 \mu \mathrm{m}$, stained with hematoxylin and eosin, were microdissected by a proficient pathologist according to a procedure based on the previously described by Ramal et al. [34], but with some modifications. An insulin needle was used to microdissect tumor cells (approx. 500 cells) which were then incubated in $100 \mu$ l of digestion buffer containing $1 \times$ standard Taq reaction buffer, $0.1 \mathrm{mg} / \mathrm{ml}$ proteinase $\mathrm{K}$ and $1 \%$ Tween-20 for $2 \mathrm{~h}$ at $56^{\circ} \mathrm{C}$. Proteinase $\mathrm{K}$ was heat-inactivated by incubating at $95^{\circ} \mathrm{C}$ for $10 \mathrm{~min}$. After centrifugation for $10 \mathrm{~min}$ at $4^{\circ} \mathrm{C}$ to remove debris, the digestion product was aliquoted and frozen at $-20^{\circ} \mathrm{C}$ until its use. Normal DNA from PBMCs was purified by using a DNA easy blood isolation kit (MO BIO Laboratories, Inc., Carlsbad, Calif., USA) according to the manufacturer's instructions. 


\section{Amplification of Microsatellites}

As was previously published [35], a panel of 11 short tandem repeat (STR) markers was selected to define HLA and $\beta_{2} \mathrm{~m} \mathrm{LOH}$ based upon their degree of polymorphism [36]. Eight STR markers are located in the short arm of chromosome 6. Seven hybridized in the region $6 \mathrm{p} 21.3$ where the genes coding for HLA are located (D6S291, D6S1618, D6S1583, D6S273, D6S265, D6S105 and D6S276). One is telomeric to the short arm of chromosome 6 at 6 p25 (D6S1617). To define whether or not LOH is caused by total chromosome loss, we included a STR (D6S311) located on the long arm of chromosome 6 . Two lasting STRs are located on chromosome 15 (D15S209 and D15S126), and flank the $\beta_{2}$ m locus in the $15 \mathrm{q} 21$ region. The forward primers were stained with two different fluorochromes: 6-FAM in D6S311, D6S291, D6S265, D6S105, D15S126, and D15S209 markers, and TET in D6S1618, D6S273, D6S1583, D6S1617 and D6S276 markers. They were then used in STR analysis.

\section{LOH Analysis}

Polymerase chain reaction (PCR) and capillary electrophoresis for $\mathrm{LOH}$ analysis were performed as previously described in the 14th International Histocompatibility Workshop, 2002 [http://www.microbiol.unimelb.edu.au/14ihiws/]. Briefly, the PCR was performed in a final volume of $25 \mu$ l of reaction mixture containing $1 \times$ Taq polymerase buffer, $1-4 \mathrm{pmol}$ of each primer pair, $2 \mathrm{mM}$ dNTP, $1.5 \mathrm{mM} \mathrm{MgCl}_{2}, 0.25 \mathrm{U}$ Taq polymerase, and $200 \mathrm{ng}$ of normal DNA obtained from PBMCs (no tumor DNA) or DNA obtained from tumor cells. The thermal cycling profile, using a PTC-100 Cycler (MJ Research, Inc.) was as follows: an initial denaturating step at $96^{\circ} \mathrm{C}$ for $5 \mathrm{~min}, 33$ cycles of amplification (denaturating at $96^{\circ} \mathrm{C}$ for $1 \mathrm{~min}$, annealing at $55^{\circ} \mathrm{C}$ for $2 \mathrm{~min}$, and elongation at $72^{\circ} \mathrm{C}$ for $1 \mathrm{~min}$ ), finally, an extension step at $72^{\circ} \mathrm{C}$ for $6 \mathrm{~min}$. Afterwards, $1 \mu \mathrm{l}$ of the PCR product was added to a mix that contained $12 \mu \mathrm{l}$ of formamide and $0.5 \mu \mathrm{l}$ of internal molecular weight standard TAMRA 500. The samples were then homogenized and heat-denatured at $95^{\circ} \mathrm{C}$ for $3 \mathrm{~min}$. Capillary electrophoresis was performed using the polymer POP4 for $30 \mathrm{~min}$ at $60^{\circ} \mathrm{C}$ using an ABI PRISM ${ }^{\circledR} 310$ DNA sequencer (Applied Biosystems, Foster City, Calif., USA).

The analysis was performed using GeneScan ${ }^{\circledR}$ analysis software (Applied Biosystems), and the alleles were assigned with Genotyper software (Applied Biosystems). STR markers were informative when the PCR product obtained from the normal DNA of PBMCs showed two different alleles corresponding to a heterozygous individual. LOH was assigned when a signal reduction of more than $25 \%$ in one allele was observed in the tumor sample compared to the same STR marker in the control sample [34]. Haplotype loss was assigned when a tumor sample showed $\mathrm{LOH}$ in three or more STR markers [34, 37].

$\mathrm{LOH}$ was calculated as:

height of tumor allele $2 /$ height of tumor allele 1

height of normal allele 2 /height of normal allele 1

where allele 2 is the long allele and allele 1 is the short one.

\section{Antibodies and Reagents}

Immunohistochemistry study was performed using the following monoclonal antibodies (mAbs): W6/32 (1:100 dilution in PBS $1 \times$ ) which recognizes a determinant expressed on the heavy chain of $\beta_{2}$ m-associated HLA-A, -B, and -C (BD Pharmingen,
San Diego, Calif., USA), E10 (1:100 dilution in PBS 1×), IgG2a, anti-human IL-10 mAb (Santa Cruz Biotechnology, Santa Cruz, Calif., USA), and 4H84 (1:500 dilution in PBS $1 \times$ ), IgG1, antidenatured HLA-G $\alpha 1$ domain of the heavy chain (BD Pharmingen). Immunocomplex detection was performed using the DakoCytomation LSAB2 System HRP kit (Dako, Carpinteria, Calif., USA).

\section{Immunohistochemistry}

Immunohistochemistry analysis was performed on 63 frozen cervical biopsies. Briefly, $4 \mu \mathrm{m}$-thick cryostat sections were cut and placed on glass slides. After $15 \mathrm{~min}$ of air-drying, the sections were fixed for $10 \mathrm{~min}$ in ice-cold acetone and stored at $-20^{\circ} \mathrm{C}$ until its use. Non-specific binding sites were blocked by incubating slides with $20 \% \mathrm{AB}$ serum/PBS for at least $15 \mathrm{~min}$ at room temperature. Endogenous peroxidase activity was blocked by incubation with $3 \%$ hydrogen peroxide for $15 \mathrm{~min}$. The slides were then incubated for $30 \mathrm{~min}$ at room temperature with the corresponding dilution of anti-HLA class I mAb (W6/32), anti-IL-10 mAb (E10) or anti-HLA-G mAb (4H84). Incubation with PBS was used as a negative control. After three washes with $1 \times$ PBS, biotinylated goat anti-mouse immunoglobulins (Dako) were applied and incubated at room temperature for $30 \mathrm{~min}$. The slides were then incubated with an avidin-biotin-peroxidase conjugate for $30 \mathrm{~min}$. The immunocomplexes were developed with a fresh 3,3'-diaminobenzidine tetrahydrochloride solution. The slides were counterstained with hematoxylin, dehydrated in alcohol, and cleared in xylene before mounting.

The staining intensity of HLA class I expression in the tumor cells was scored as described by Feenstra et al. [36]. At normal HLA class I expression, staining of the tumor cells showed the same intensity as the surrounding stromal cells, at weak HLA class I expression, staining of the tumor cells was significantly less compared to staining of the surrounding stromal cells, and when negative, no staining of the tumor cells was observed. Necrotic or keratinized tumor cells were not taken into consideration. To analyze HLA-G expression, sections of cytotrophoblast from human placenta served as an HLA-G-positive control. HLA-G expression was graded as follows: negative, 1 for focal expression or weak expression, 2 for $>75 \%$ of expression.

\section{Statistical Interpretation}

Statistical analysis was performed using SPSS 18.0 software. The percentage of heterozygosity for individual STR markers was calculated as the ratio of normal peripheral blood samples that were heterozygous to the total population analyzed. The percentage of $\mathrm{LOH}$ was calculated by applying the formula described above only for those individuals whose peripheral blood was heterozygous. $\chi^{2}$ test or, where appropriate, Fisher's exact test was used to assess differences in LOH frequency and correlations between HLA class I, HLA-G and IL-10 expression. The survival rates were calculated by the Kaplan-Meier method, and the differences between the survival curves were determined by the logrank test. Overall survival was defined as the interval from the beginning of treatment to death or last visit date. For all tests, $p$ values $<0.05$ were considered statistically significant. 


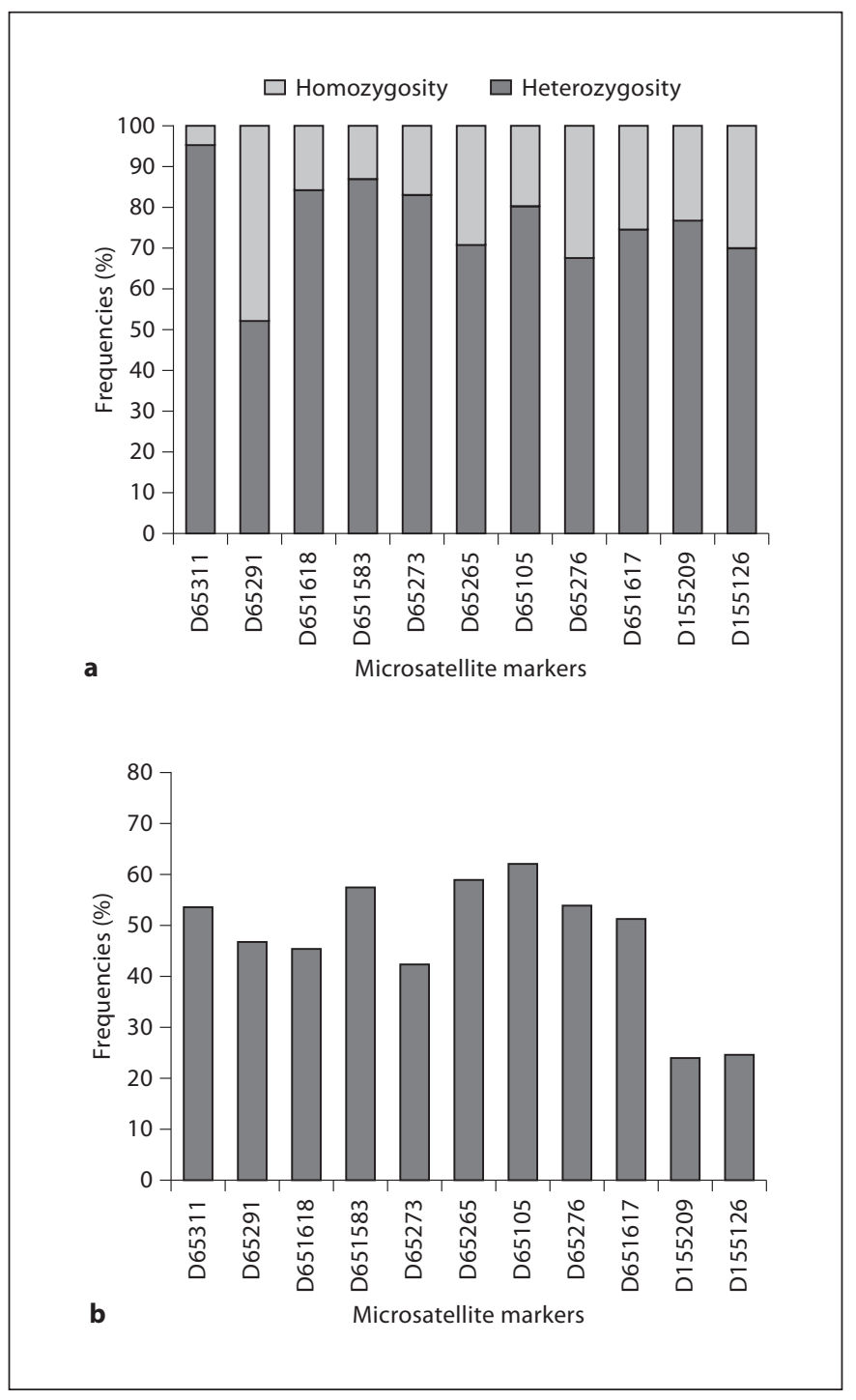

Fig. 1. Percentage of heterozygosity (a) and LOH frequencies (b) of eleven microsatellite markers in 63 microdissected tumor cells and normal cells from cervical cancer patients.

\section{Results}

\section{Clinical Characteristics of Patients}

The clinical characteristics of the study subjects are listed in table 1 . A total of 63 female patients with a diagnosis of CIN III and invasive cervical cancer stage IBIIVB were enrolled in this study. The age range was between 25 and 63 years and the mean age of the patients was 43.08 years $( \pm 10.81 \mathrm{SD})$. Tumor sizes before treatment ranged from 1.0 to $12 \mathrm{~cm} .13$ patients $(20.6 \%)$ underwent surgical treatment, 5 patients $(7.9 \%)$ were treated
Table 1. Clinicopathological characteristics of cervical cancer patients

\begin{tabular}{llc}
\hline Age, years & & $43.08(25-63)$ \\
\hline Tumor size, cm & Before treatment & $6.09(1-12)$ \\
& After treatment & $1.47(0-6)$ \\
\hline FIGO stage & CIN III & 9 \\
& IBI-II & 10 \\
& IIA-B & 20 \\
& IIIB & 21 \\
& IVB & 3 \\
\hline HPV DNA & Simple infection & \\
& HPV 16 & $57 / 63(90.4 \%)$ \\
& HPV 33 & $1 / 63(4.7 \%)$ \\
& HPV 52 $53.5 \%)$ \\
& Multiple Infection & \\
& HPV 18 and 53 or & $2 / 63(3.0 \%)$ \\
\hline
\end{tabular}

with surgery and radiotherapy, $26(41.3 \%)$ received combined chemoradiotherapy, and 12 patients (19\%) received only radiotherapy. 7 (11.1\%) patients did not accept any therapy. The mean follow-up time was 38.93 months, with a time range from 5 to 74 months. All samples were positive for HPV DNA. The most prevalent HPV type was 16. Other, less frequent HPV types were 18, 53, 33, 52, and 58 . Only $3 \%(2 / 63)$ had a multiple infection (table 1$)$.

\section{Analysis of LOH in 6p21.3 and 15q21 and HLA Class I Expression \\ LOH was analyzed in 63 cases. Four different patterns} were found with STR analysis: partial $\mathrm{LOH}$, total $\mathrm{LOH}$, retention of heterozygosis, and homozygosis. Partial LOH was defined when one STR marker in the tumor sample had a signal intensity $\leq 25 \%$ of that observed in the same STR marker in the control sample. Total LOH was defined as the presence of only one STR allele in the tumor sample. Retention of heterozygosis was defined when the two alleles of STR amplified in the microdissected tumor cells showed a similar profile to that of the normal peripheral blood sample. Finally, homozygosis was defined as the presence of only one allele signal in the control samples. In this case, the marker was not informative. Homozygosis was observed in $19.6 \%$ of cases, while the percentage of heterozygosis varied from 51.8 to $86.4 \%$ depending on the marker (fig. 1a). LOH frequencies in the HLA region varied from 24.1 to $62 \%$ depending on the STR analyzed (fig. 1b). The total $\mathrm{LOH}$ in the $6 \mathrm{p} 21.3$ region was $81 \%$ (51 out of 63 cases), while in the 15q region, 31.3\% (15 out of 
48 cases) were found to have $\mathrm{LOH}$. When we analyzed the LOH according to cervical cancer stage (fig. 2a), a high frequency of $\mathrm{LOH}$ was observed in more advanced cervical cancer, but only the D6S105 marker showed a significant variation between different stages (Pearson's $\chi^{2}, \mathrm{p}=$ 0.044). Haplotype loss was defined in 6p21.3 when more than three consecutive microsatellite markers were lost. Haplotype loss was found in $62.7 \%$ (32 out of 51 cases). It was higher in patients with advanced cervical cancer than in those with early stages (fig. 2b).

The HLA class I expression was analyzed in 59 cases. Of these cases, $15.2 \%(9 / 59)$ showed normal HLA class I expression and were used as an internal control of HLA class I expression. Of the 50 remaining cases, $22 \%(11 / 50)$ showed a weak expression and 78\% (39/50) displayed a total loss of HLA class I expression (fig. 3a-d). Although an association between decrease of HLA class I expression and $\mathrm{LOH}$ in the $6 \mathrm{p} 21.3$ and $15 \mathrm{q}$ regions has been observed in other cancer models, in this study no significant difference in the frequency of $\mathrm{LOH}$ was observed among patients who had an alteration in HLA class I expression. Table 2 shows the analysis of LOH in 6p21.3 and 15q21 regions in cervical cancer patients with normal HLA class I expression. In the $6 \mathrm{p} 21.3$ region, $33.3 \%$ (3/9) did not show LOH, $11.1 \%$ (1/9) presented $\mathrm{LOH}$ and $55.6 \%$ (5/9) had haplotype loss. Among the patients who had altered HLA class I expression (table 3), 18.0\% (9/50) did not show $\mathrm{LOH}, 32.0 \%(16 / 50)$ presented $\mathrm{LOH}$ and $50 \%(25 / 50)$ had haplotype loss (Pearson's $\chi^{2}, \mathrm{p}=0.353$ ). When the expression of HLA class I was analyzed in relation to the $15 \mathrm{q} \mathrm{LOH}$, in patients who had a normal expression of HLA class I, 44.4\% (4/9) did not have LOH and 55.6\% (5/9) showed LOH. Among patients who had altered HLA class I expression, $74.3 \%(26 / 35)$ did not have $\mathrm{LOH}$ and $25.7 \%(9 / 35)$ showed LOH (Fisher's exact test, $\mathrm{p}=0.097$ ).

\section{Association of IL-10 Expression and Downregulation}

of HLA Class I Expression

It has been suggested that IL-10 may negatively regulate HLA class I expression at the tumor cell surface, thus allowing the escape from tumor cell lysis mediated by CTLs. In this study, a significant association was found between IL-10 expression and decrease of HLA class I expression (Pearson's $\chi^{2}, \mathrm{p}=0.028$ ). Among the patients who expressed IL-10 cytokine, $48.1 \%(13 / 27)$ had a total loss of HLA class I expression, $25.9 \%$ (7/27) had weak, and $25.9 \%(7 / 27)$ had normal HLA class I expression (table 4). However, among the patients who did not have IL-10 expression, a high percentage $(80.6 \%, 25 / 31)$ was also HLA class I negative.

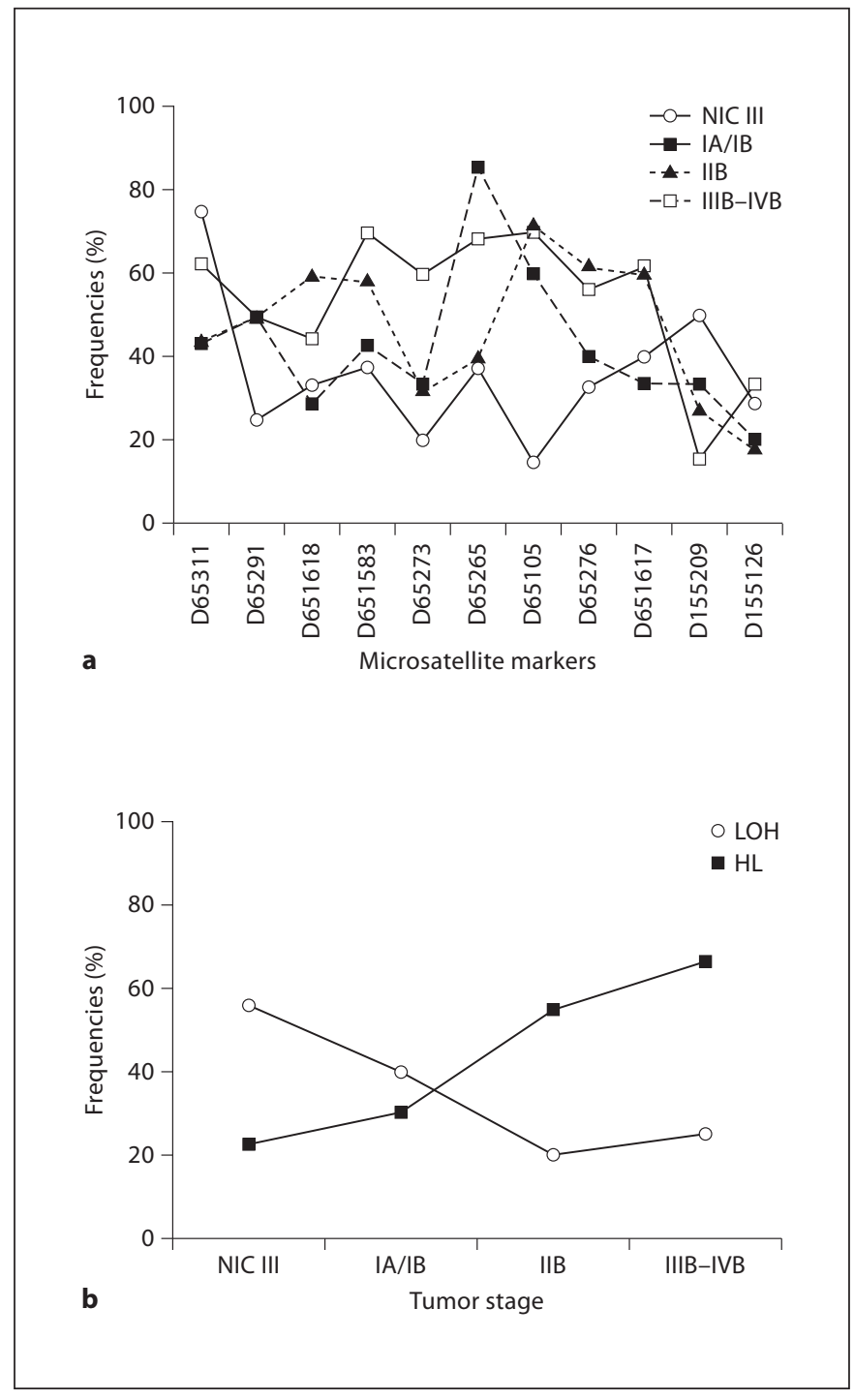

Fig. 2. $\mathrm{LOH}$ frequencies according to tumor stage of cervical cancer patients (a) and LOH and LH frequencies (b) of eleven microsatellite markers in 63 microdissected samples.

\section{IL-10 Expression Is Associated with HLA-G}

Expression in Cervical Cancer Samples

Overall, IL-10 was expressed in 46.6\% (27/58) of cases, while HLA-G protein expression was observed in $27.6 \%$ (16/58) of cases. A significant association was found between IL-10 and HLA-G expression (Fisher's exact test, $\mathrm{p}=0.000)$. In most of the HLA-G-positive cases, $14 / 16$ (87.5\%) exhibited IL-10 expression. Similarly, a high percentage $(69 \%, 29 / 42)$ of the HLA-G-negative cases did not express IL-10 (table 4). It has been reported that HLA-G expression status is associated with tumor histologic 

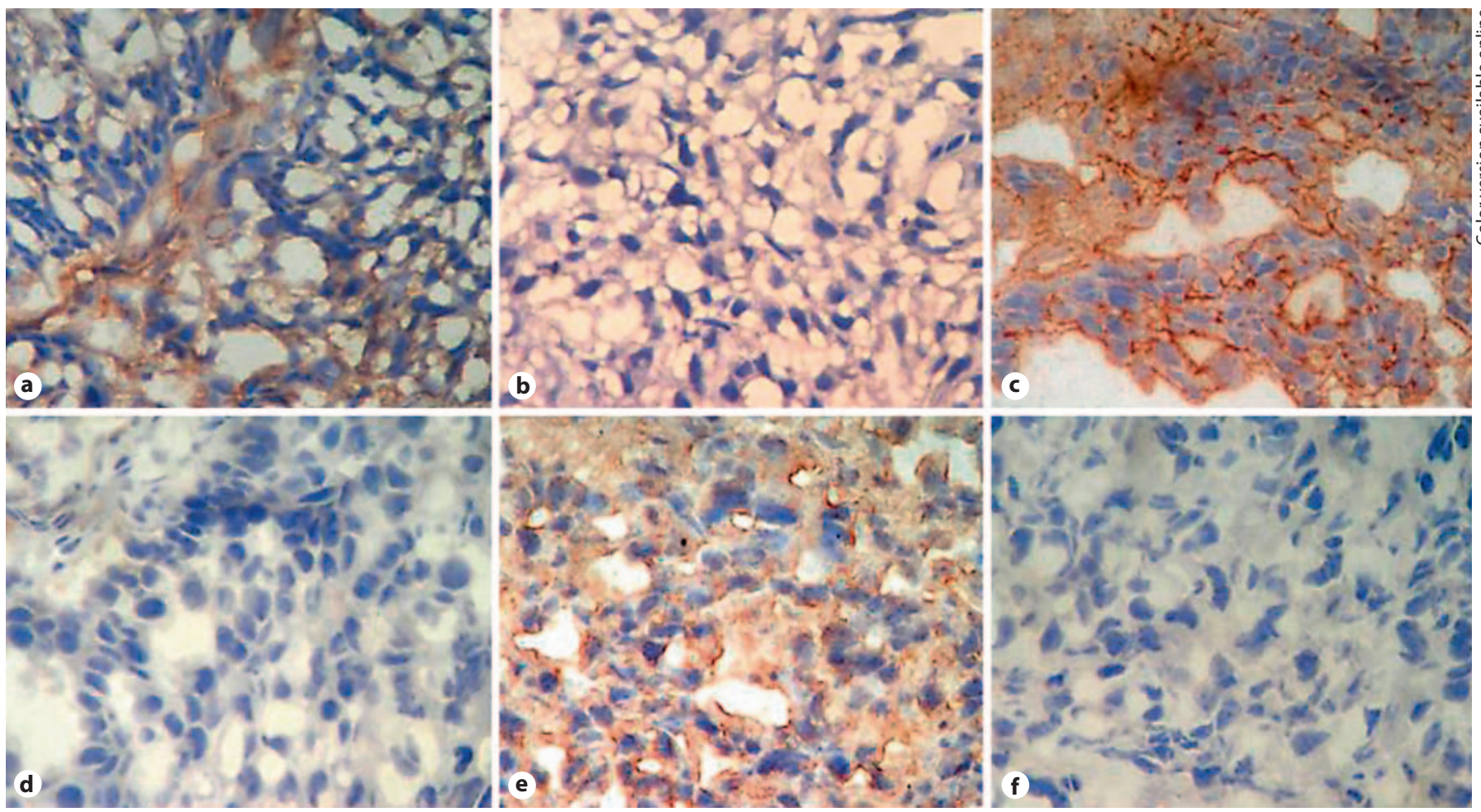

Fig. 3. Immunohistochemistry in cervical biopsies. HLA class I expression (mAb W6/32) from representative positive cervical cancer sample (a) and negative control (b). HLA-G expression (mAb 4H84) from representative positive cervical cancer sample (c) and negative control (d). Finally, IL-10 expression (mAb E10) from representative positive cervical cancer sample (c) and negative control (d).

Table 2. Analysis of LOH in 6p21.3 and 15q21 in cervical cancer patients with normal HLA class I expression

\begin{tabular}{|c|c|c|c|c|c|c|c|c|c|c|c|c|c|c|}
\hline \multirow[t]{2}{*}{ Tumor } & \multirow{2}{*}{$\begin{array}{l}\text { IHC } \\
\text { HLA-I } \\
\text { expression }\end{array}$} & \multirow{2}{*}{$\begin{array}{l}6 q \\
\text { D6S311 }\end{array}$} & \multirow{2}{*}{$\begin{array}{l}6 p 25 \\
\text { D6S1617 }\end{array}$} & \multicolumn{8}{|c|}{ LOH 6 p21 } & \multicolumn{3}{|c|}{ LOH $15 q$} \\
\hline & & & & D6S291 & D6S1618 & D6S1583 & D6S273 & D6S265 & D6S105 & D6S276 & $\begin{array}{l}\text { Total } \\
\mathrm{OH}\end{array}$ & D15S209 & D15S126 & $\begin{array}{l}\text { Total } \\
\text { LOH }\end{array}$ \\
\hline 17 & + & ND & $\mathrm{R}$ & $\mathrm{R}$ & $\mathrm{H}$ & $\mathrm{R}$ & ND & $\mathrm{R}$ & $\mathrm{H}$ & $\mathrm{R}$ & $\mathrm{R}$ & ND & $\mathrm{R}$ & $\mathrm{R}$ \\
\hline 21 & + & $\mathrm{R}$ & $\mathrm{H}$ & $\mathrm{R}$ & $\mathrm{H}$ & $\mathrm{R}$ & $\mathrm{R}$ & $\mathrm{R}$ & $\mathrm{R}$ & $\mathrm{R}$ & $\mathrm{R}$ & $\mathrm{H}$ & $\mathrm{R}$ & $\mathrm{R}$ \\
\hline 25 & + & ND & $\mathrm{R}$ & $\mathrm{H}$ & $\mathrm{R}$ & $\mathrm{R}$ & $\mathrm{R}$ & $\mathrm{H}$ & $\mathrm{R}$ & ND & $\mathrm{R}$ & ND & $\mathrm{R}$ & $\mathrm{R}$ \\
\hline 63 & + & ND & $\mathrm{R}$ & $\mathrm{R}$ & $\mathrm{R}$ & $\mathrm{L}$ & $\mathrm{R}$ & $\mathrm{R}$ & $\mathrm{R}$ & $\mathrm{R}$ & $\mathrm{L}$ & ND & $\mathrm{L}$ & $\mathrm{L}$ \\
\hline 31 & + & $\mathrm{L}$ & ND & ND & $\mathrm{L}$ & $\mathrm{L}$ & $\mathrm{R}$ & $\mathrm{L}$ & $\mathrm{L}$ & $\mathrm{L}$ & $\mathrm{HL}$ & $\mathrm{L}$ & ND & $\mathrm{L}$ \\
\hline 35 & + & $\mathrm{L}$ & ND & $\mathrm{L}$ & $\mathrm{R}$ & $\mathrm{L}$ & $\mathrm{L}$ & $\mathrm{H}$ & $\mathrm{L}$ & $\mathrm{L}$ & $\mathrm{HL}$ & $\mathrm{L}$ & $\mathrm{H}$ & $\mathrm{L}$ \\
\hline 53 & + & $\mathrm{R}$ & $\mathrm{L}$ & $\mathrm{H}$ & $\mathrm{L}$ & $\mathrm{L}$ & $\mathrm{R}$ & $\mathrm{H}$ & $\mathrm{L}$ & ND & $\mathrm{HL}$ & $\mathrm{R}$ & $\mathrm{R}$ & $\mathrm{R}$ \\
\hline 37 & + & $\mathrm{L}$ & $\mathrm{L}$ & $\mathrm{H}$ & $\mathrm{L}$ & ND & $\mathrm{L}$ & $\mathrm{H}$ & $\mathrm{L}$ & $\mathrm{H}$ & $\mathrm{HL}$ & $\mathrm{L}$ & $\mathrm{R}$ & $\mathrm{L}$ \\
\hline 41 & + & $\mathrm{L}$ & ND & $\mathrm{H}$ & $\mathrm{L}$ & $\mathrm{L}$ & $\mathrm{L}$ & $\mathrm{L}$ & $\mathrm{L}$ & $\mathrm{L}$ & $\mathrm{HL}$ & $\mathrm{H}$ & $\mathrm{L}$ & $\mathrm{L}$ \\
\hline
\end{tabular}

Immunohistochemical staining (IHC) of HLA class I (W6/32) and LOH on chromosomes 6p and 15q of 9 invasive cervical cancers. $\mathrm{R}=$ Retention (no LOH); L = LOH; HL= haplotype loss; $\mathrm{H}$ = homozygous (not informative); $\mathrm{ND}=$ not determined. 
Table 3. Analysis of LOH in 6p21.3 and 15q21 in cervical cancer patients with altered HLA class I expression

\begin{tabular}{|c|c|c|c|c|c|c|c|c|c|c|c|c|c|c|}
\hline \multirow[t]{2}{*}{ Tumor } & \multirow{2}{*}{$\begin{array}{l}\text { IHC } \\
\text { HLA-I }\end{array}$} & \multirow{2}{*}{$\begin{array}{l}6 q \\
\text { D6S311 }\end{array}$} & \multirow{2}{*}{$\begin{array}{l}6 \mathrm{p} 25 \\
\text { D6S1617 }\end{array}$} & \multicolumn{8}{|c|}{ LOH $6 p$} & \multicolumn{3}{|c|}{$\mathrm{LOH} 15 \mathrm{q}$} \\
\hline & & & & D6S291 & D6S1618 & D6S1583 & D6S273 & D6S265 & D6S105 & D6S276 & $\begin{array}{l}\text { Total } \\
\text { LOH }\end{array}$ & D15S209 & D15S126 & $\begin{array}{l}\text { Total } \\
\text { LOH }\end{array}$ \\
\hline 54 & - & $\mathrm{R}$ & ND & $\mathrm{H}$ & $\mathrm{R}$ & $\mathrm{R}$ & $\mathrm{R}$ & $\mathrm{H}$ & $\mathrm{R}$ & $\mathrm{H}$ & $\mathrm{R}$ & ND & $\mathrm{R}$ & $\mathrm{R}$ \\
\hline 23 & - & $\mathrm{R}$ & $\mathrm{H}$ & $\mathrm{R}$ & $\mathrm{R}$ & $\mathrm{R}$ & $\mathrm{R}$ & $\mathrm{H}$ & $\mathrm{H}$ & $\mathrm{R}$ & $\mathrm{R}$ & $\mathrm{R}$ & $\mathrm{R}$ & $\mathrm{R}$ \\
\hline 13 & - & ND & $\mathrm{R}$ & $\mathrm{H}$ & $\mathrm{R}$ & $\mathrm{R}$ & $\mathrm{R}$ & $\mathrm{H}$ & $\mathrm{H}$ & $\mathrm{R}$ & $\mathrm{R}$ & ND & $\mathrm{R}$ & $\mathrm{R}$ \\
\hline 22 & - & ND & $\mathrm{L}$ & $\mathrm{R}$ & $\mathrm{R}$ & $\mathrm{R}$ & $\mathrm{R}$ & $\mathrm{R}$ & $\mathrm{H}$ & $\mathrm{H}$ & $\mathrm{R}$ & ND & $\mathrm{R}$ & $\mathrm{R}$ \\
\hline 8 & - & $\mathrm{H}$ & $\mathrm{R}$ & $\mathrm{H}$ & $\mathrm{H}$ & $\mathrm{H}$ & $\mathrm{R}$ & $\mathrm{R}$ & $\mathrm{R}$ & $\mathrm{R}$ & $\mathrm{R}$ & ND & $\mathrm{R}$ & $\mathrm{R}$ \\
\hline 38 & - & ND & $\mathrm{H}$ & $\mathrm{R}$ & $\mathrm{R}$ & $\mathrm{R}$ & $\mathrm{R}$ & $\mathrm{R}$ & $\mathrm{R}$ & $\mathrm{R}$ & $\mathrm{R}$ & ND & ND & ND \\
\hline 7 & - & ND & ND & $\mathrm{H}$ & $\mathrm{R}$ & $\mathrm{R}$ & $\mathrm{R}$ & $\mathrm{R}$ & $\mathrm{H}$ & ND & $\mathrm{R}$ & ND & $\mathrm{H}$ & ND \\
\hline 11 & - & ND & $\mathrm{R}$ & $\mathrm{R}$ & $\mathrm{R}$ & $\mathrm{R}$ & $\mathrm{H}$ & $\mathrm{H}$ & $\mathrm{R}$ & $\mathrm{R}$ & $\mathrm{R}$ & $\mathrm{L}$ & ND & $\mathrm{L}$ \\
\hline 26 & - & $\mathrm{R}$ & $\mathrm{R}$ & $\mathrm{R}$ & $\mathrm{R}$ & $\mathrm{R}$ & $\mathrm{R}$ & $\mathrm{R}$ & $\mathrm{R}$ & $\mathrm{R}$ & $\mathrm{R}$ & $\mathrm{H}$ & $\mathrm{L}$ & $\mathrm{L}$ \\
\hline 3 & - & ND & $\mathrm{R}$ & $\mathrm{R}$ & $\mathrm{R}$ & $\mathrm{R}$ & ND & $\mathrm{L}$ & $\mathrm{R}$ & $\mathrm{L}$ & $\mathrm{L}$ & ND & ND & ND \\
\hline 4 & - & $\mathrm{L}$ & $\mathrm{R}$ & $\mathrm{R}$ & $\mathrm{R}$ & $\mathrm{L}$ & ND & $\mathrm{R}$ & $\mathrm{R}$ & ND & $\mathrm{L}$ & ND & $\mathrm{L}$ & $\mathrm{L}$ \\
\hline 61 & - & $\mathrm{L}$ & $\mathrm{R}$ & $\mathrm{R}$ & $\mathrm{R}$ & $\mathrm{H}$ & $\mathrm{R}$ & $\mathrm{R}$ & $\mathrm{L}$ & $\mathrm{H}$ & $\mathrm{L}$ & ND & $\mathrm{R}$ & $\mathrm{R}$ \\
\hline 30 & - & ND & ND & $\mathrm{R}$ & ND & $\mathrm{R}$ & $\mathrm{R}$ & $\mathrm{R}$ & $\mathrm{L}$ & $\mathrm{H}$ & $\mathrm{L}$ & $\mathrm{R}$ & $\mathrm{H}$ & $\mathrm{R}$ \\
\hline 34 & - & ND & ND & $\mathrm{H}$ & ND & $\mathrm{L}$ & $\mathrm{R}$ & $\mathrm{H}$ & $\mathrm{L}$ & $\mathrm{R}$ & $\mathrm{L}$ & $\mathrm{H}$ & ND & ND \\
\hline 55 & - & $\mathrm{R}$ & $\mathrm{R}$ & $\mathrm{H}$ & L & $\mathrm{H}$ & $\mathrm{R}$ & $\mathrm{R}$ & $\mathrm{H}$ & $\mathrm{R}$ & $\mathrm{L}$ & $\mathrm{R}$ & $\mathrm{H}$ & $\mathrm{R}$ \\
\hline 15 & - & $\mathrm{R}$ & $\mathrm{R}$ & $\mathrm{H}$ & $\mathrm{R}$ & $\mathrm{L}$ & $\mathrm{R}$ & $\mathrm{L}$ & $\mathrm{R}$ & ND & $\mathrm{L}$ & $\mathrm{R}$ & $\mathrm{R}$ & $\mathrm{R}$ \\
\hline 33 & - & $\mathrm{R}$ & $\mathrm{R}$ & $\mathrm{H}$ & $\mathrm{R}$ & $\mathrm{L}$ & $\mathrm{R}$ & $\mathrm{R}$ & $\mathrm{R}$ & $\mathrm{R}$ & $\mathrm{L}$ & $\mathrm{R}$ & $\mathrm{R}$ & $\mathrm{R}$ \\
\hline 40 & - & $\mathrm{R}$ & $\mathrm{R}$ & $\mathrm{R}$ & $\mathrm{R}$ & $\mathrm{R}$ & $\mathrm{H}$ & $\mathrm{L}$ & $\mathrm{R}$ & $\mathrm{R}$ & L & $\mathrm{L}$ & ND & $\mathrm{L}$ \\
\hline 32 & $\mathrm{~W}$ & $\mathrm{R}$ & ND & ND & $\mathrm{L}$ & $\mathrm{R}$ & $\mathrm{H}$ & $\mathrm{R}$ & $\mathrm{R}$ & $\mathrm{H}$ & $\mathrm{L}$ & $\mathrm{R}$ & ND & $\mathrm{R}$ \\
\hline 5 & W & $\mathrm{L}$ & $\mathrm{R}$ & $\mathrm{H}$ & $\mathrm{L}$ & $\mathrm{H}$ & $\mathrm{H}$ & $\mathrm{H}$ & $\mathrm{H}$ & $\mathrm{L}$ & $\mathrm{L}$ & $\mathrm{H}$ & $\mathrm{H}$ & $\mathrm{H}$ \\
\hline 6 & $\mathrm{~W}$ & ND & ND & $\mathrm{H}$ & $\mathrm{L}$ & $\mathrm{R}$ & $\mathrm{H}$ & $\mathrm{H}$ & $\mathrm{R}$ & ND & $\mathrm{L}$ & ND & $\mathrm{R}$ & $\mathrm{R}$ \\
\hline 59 & $\mathrm{~W}$ & $\mathrm{~L}$ & ND & $\mathrm{H}$ & $\mathrm{R}$ & $\mathrm{R}$ & $\mathrm{R}$ & $\mathrm{H}$ & $\mathrm{L}$ & $\mathrm{R}$ & $\mathrm{L}$ & $\mathrm{R}$ & $\mathrm{H}$ & $\mathrm{R}$ \\
\hline 46 & W & $\mathrm{R}$ & $\mathrm{R}$ & $\mathrm{H}$ & $\mathrm{R}$ & $\mathrm{R}$ & $\mathrm{R}$ & $\mathrm{R}$ & $\mathrm{L}$ & $\mathrm{R}$ & $\mathrm{L}$ & ND & $\mathrm{R}$ & $\mathrm{R}$ \\
\hline 12 & W & $\mathrm{R}$ & $\mathrm{L}$ & $\mathrm{H}$ & $\mathrm{R}$ & $\mathrm{R}$ & $\mathrm{R}$ & $\mathrm{L}$ & $\mathrm{H}$ & $\mathrm{H}$ & $\mathrm{L}$ & $\mathrm{R}$ & $\mathrm{L}$ & $\mathrm{L}$ \\
\hline 49 & W & $\mathrm{R}$ & $\mathrm{L}$ & ND & ND & $\mathrm{H}$ & $\mathrm{L}$ & $\mathrm{L}$ & $\mathrm{H}$ & $\mathrm{H}$ & $\mathrm{L}$ & ND & ND & ND \\
\hline 47 & $\mathrm{~W}$ & $\mathrm{R}$ & $\mathrm{H}$ & $\mathrm{L}$ & $\mathrm{L}$ & $\mathrm{H}$ & $\mathrm{L}$ & $\mathrm{L}$ & $\mathrm{L}$ & $\mathrm{L}$ & HL & $\mathrm{H}$ & $\mathrm{H}$ & $\mathrm{H}$ \\
\hline 44 & W & $\mathrm{R}$ & $\mathrm{L}$ & $\mathrm{H}$ & $\mathrm{L}$ & $\mathrm{L}$ & $\mathrm{L}$ & $\mathrm{L}$ & $\mathrm{L}$ & $\mathrm{L}$ & HL & ND & $\mathrm{H}$ & ND \\
\hline 28 & $\mathrm{~W}$ & $\mathrm{~L}$ & $\mathrm{~L}$ & $\mathrm{R}$ & $\mathrm{L}$ & $\mathrm{L}$ & $\mathrm{L}$ & $\mathrm{L}$ & $\mathrm{L}$ & $\mathrm{L}$ & HL & ND & ND & ND \\
\hline 45 & $\mathrm{~W}$ & ND & ND & $\mathrm{H}$ & $\mathrm{L}$ & $\mathrm{R}$ & $\mathrm{L}$ & $\mathrm{L}$ & $\mathrm{R}$ & $\mathrm{H}$ & HL & $\mathrm{H}$ & $\mathrm{H}$ & $\mathrm{H}$ \\
\hline 2 & - & $\mathrm{L}$ & $\mathrm{L}$ & $\mathrm{H}$ & ND & $\mathrm{L}$ & $\mathrm{L}$ & $\mathrm{L}$ & $\mathrm{H}$ & $\mathrm{H}$ & HL & ND & $\mathrm{L}$ & $\mathrm{L}$ \\
\hline 10 & - & $\mathrm{R}$ & $\mathrm{R}$ & $\mathrm{L}$ & $\mathrm{L}$ & $\mathrm{H}$ & $\mathrm{R}$ & $\mathrm{L}$ & $\mathrm{L}$ & $\mathrm{L}$ & HL & ND & $\mathrm{R}$ & $\mathrm{R}$ \\
\hline 18 & - & $\mathrm{R}$ & $\mathrm{H}$ & $\mathrm{H}$ & $\mathrm{L}$ & $\mathrm{L}$ & $\mathrm{R}$ & $\mathrm{L}$ & $\mathrm{L}$ & $\mathrm{H}$ & HL & ND & $\mathrm{R}$ & $\mathrm{R}$ \\
\hline 20 & - & $\mathrm{L}$ & $\mathrm{H}$ & L & L & L & $\mathrm{H}$ & $\mathrm{L}$ & $\mathrm{L}$ & $\mathrm{L}$ & HL & $\mathrm{R}$ & $\mathrm{H}$ & $\mathrm{H}$ \\
\hline 24 & - & $\mathrm{R}$ & $\mathrm{R}$ & $\mathrm{L}$ & $\mathrm{L}$ & $\mathrm{L}$ & $\mathrm{L}$ & $\mathrm{H}$ & $\mathrm{L}$ & $\mathrm{L}$ & HL & $\mathrm{H}$ & $\mathrm{R}$ & $\mathrm{R}$ \\
\hline 27 & - & $\mathrm{L}$ & ND & $\mathrm{L}$ & $\mathrm{H}$ & $\mathrm{L}$ & $\mathrm{H}$ & $\mathrm{H}$ & $\mathrm{L}$ & $\mathrm{L}$ & HL & $\mathrm{H}$ & $\mathrm{L}$ & $\mathrm{L}$ \\
\hline 29 & - & $\mathrm{L}$ & $\mathrm{L}$ & $\mathrm{L}$ & $\mathrm{L}$ & $\mathrm{H}$ & $\mathrm{L}$ & $\mathrm{H}$ & $\mathrm{L}$ & $\mathrm{L}$ & HL & $\mathrm{R}$ & $\mathrm{H}$ & $\mathrm{H}$ \\
\hline 36 & - & $\mathrm{R}$ & $\mathrm{L}$ & $\mathrm{L}$ & $\mathrm{H}$ & ND & $\mathrm{H}$ & $\mathrm{H}$ & $\mathrm{L}$ & $\mathrm{L}$ & HL & $\mathrm{R}$ & ND & $\mathrm{R}$ \\
\hline 42 & - & $\mathrm{H}$ & $\mathrm{H}$ & $\mathrm{L}$ & ND & $\mathrm{L}$ & $\mathrm{L}$ & $\mathrm{H}$ & $\mathrm{L}$ & ND & HL & ND & ND & ND \\
\hline 43 & - & $\mathrm{L}$ & $\mathrm{L}$ & $\mathrm{H}$ & $\mathrm{R}$ & $\mathrm{L}$ & $\mathrm{L}$ & $\mathrm{L}$ & $\mathrm{L}$ & $\mathrm{H}$ & $\mathrm{HL}$ & $\mathrm{R}$ & $\mathrm{L}$ & $\mathrm{L}$ \\
\hline 48 & - & $\mathrm{L}$ & $\mathrm{L}$ & $\mathrm{H}$ & $\mathrm{L}$ & $\mathrm{L}$ & $\mathrm{L}$ & $\mathrm{L}$ & $\mathrm{L}$ & $\mathrm{L}$ & HL & $\mathrm{L}$ & ND & $\mathrm{L}$ \\
\hline 56 & - & $\mathrm{L}$ & $\mathrm{H}$ & $\mathrm{L}$ & $\mathrm{H}$ & $\mathrm{H}$ & $\mathrm{L}$ & $\mathrm{L}$ & $\mathrm{L}$ & $\mathrm{L}$ & HL & $\mathrm{R}$ & $\mathrm{H}$ & $\mathrm{R}$ \\
\hline 58 & - & ND & $\mathrm{L}$ & $\mathrm{L}$ & $\mathrm{H}$ & $\mathrm{L}$ & $\mathrm{H}$ & $\mathrm{L}$ & $\mathrm{L}$ & $\mathrm{L}$ & HL & $\mathrm{R}$ & ND & $\mathrm{R}$ \\
\hline 60 & - & $\mathrm{H}$ & $\mathrm{L}$ & $\mathrm{R}$ & $\mathrm{R}$ & $\mathrm{L}$ & $\mathrm{L}$ & $\mathrm{H}$ & $\mathrm{H}$ & $\mathrm{L}$ & HL & $\mathrm{R}$ & ND & $\mathrm{R}$ \\
\hline 62 & - & $\mathrm{L}$ & $\mathrm{L}$ & $\mathrm{H}$ & $\mathrm{H}$ & $\mathrm{L}$ & $\mathrm{L}$ & $\mathrm{L}$ & $\mathrm{L}$ & $\mathrm{H}$ & HL & $\mathrm{R}$ & $\mathrm{R}$ & $\mathrm{R}$ \\
\hline 16 & - & $\mathrm{L}$ & $\mathrm{H}$ & $\mathrm{L}$ & ND & $\mathrm{L}$ & ND & $\mathrm{L}$ & $\mathrm{L}$ & $\mathrm{H}$ & HL & ND & ND & $\mathrm{ND}$ \\
\hline 39 & - & $\mathrm{L}$ & $\mathrm{H}$ & $\mathrm{H}$ & $\mathrm{L}$ & $\mathrm{L}$ & $\mathrm{R}$ & $\mathrm{L}$ & $\mathrm{L}$ & $\mathrm{L}$ & HL & $\mathrm{R}$ & $\mathrm{H}$ & $\mathrm{R}$ \\
\hline 50 & - & ND & $\mathrm{L}$ & $\mathrm{L}$ & $\mathrm{R}$ & $\mathrm{L}$ & ND & $\mathrm{H}$ & $\mathrm{L}$ & ND & HL & ND & $\mathrm{R}$ & $\mathrm{R}$ \\
\hline 52 & - & $\mathrm{L}$ & ND & $\mathrm{L}$ & ND & $\mathrm{L}$ & $\mathrm{L}$ & $\mathrm{R}$ & ND & $\mathrm{H}$ & HL & $\mathrm{R}$ & ND & $\mathrm{R}$ \\
\hline 14 & - & $\mathrm{L}$ & $\mathrm{L}$ & $\mathrm{H}$ & $\mathrm{R}$ & $\mathrm{L}$ & $\mathrm{L}$ & $\mathrm{L}$ & $\mathrm{L}$ & ND & HL & ND & $\mathrm{H}$ & ND \\
\hline 51 & - & $\mathrm{L}$ & $\mathrm{L}$ & $\mathrm{R}$ & $\mathrm{L}$ & $\mathrm{L}$ & $\mathrm{H}$ & $\mathrm{L}$ & $\mathrm{R}$ & $\mathrm{L}$ & HL & $\mathrm{R}$ & $\mathrm{R}$ & $\mathrm{R}$ \\
\hline 1 & ND & $\mathrm{R}$ & $\mathrm{L}$ & $\mathrm{H}$ & $\mathrm{L}$ & $\mathrm{R}$ & $\mathrm{R}$ & $\mathrm{L}$ & $\mathrm{L}$ & $\mathrm{R}$ & HL & $\mathrm{R}$ & $\mathrm{R}$ & $\mathrm{R}$ \\
\hline 57 & ND & ND & $\mathrm{H}$ & $\mathrm{H}$ & ND & $\mathrm{L}$ & $\mathrm{L}$ & $\mathrm{L}$ & ND & $\mathrm{R}$ & HL & $\mathrm{R}$ & ND & $\mathrm{R}$ \\
\hline 9 & ND & ND & $\mathrm{H}$ & $\mathrm{L}$ & $\mathrm{R}$ & $\mathrm{R}$ & $\mathrm{R}$ & $\mathrm{R}$ & $\mathrm{R}$ & $\mathrm{H}$ & $\mathrm{L}$ & ND & $\mathrm{R}$ & $\mathrm{R}$ \\
\hline 19 & ND & $\mathrm{L}$ & $\mathrm{H}$ & $\mathrm{R}$ & ND & $\mathrm{L}$ & $\mathrm{H}$ & $\mathrm{R}$ & $\mathrm{R}$ & $\mathrm{H}$ & $\mathrm{L}$ & $\mathrm{L}$ & $\mathrm{R}$ & $\mathrm{L}$ \\
\hline
\end{tabular}

Immunohistochemical staining (IHC) of HLA class I (W6/32) and LOH on chromosomes 6p and 15q of 54 invasive cervical cancers. $\mathrm{R}=$ Retention (no $\mathrm{LOH}$ ); $\mathrm{L}=\mathrm{LOH} ; \mathrm{HL}=$ haplotype loss; $\mathrm{H}=$ homozygous (not informative); $\mathrm{ND}=$ not determined. 


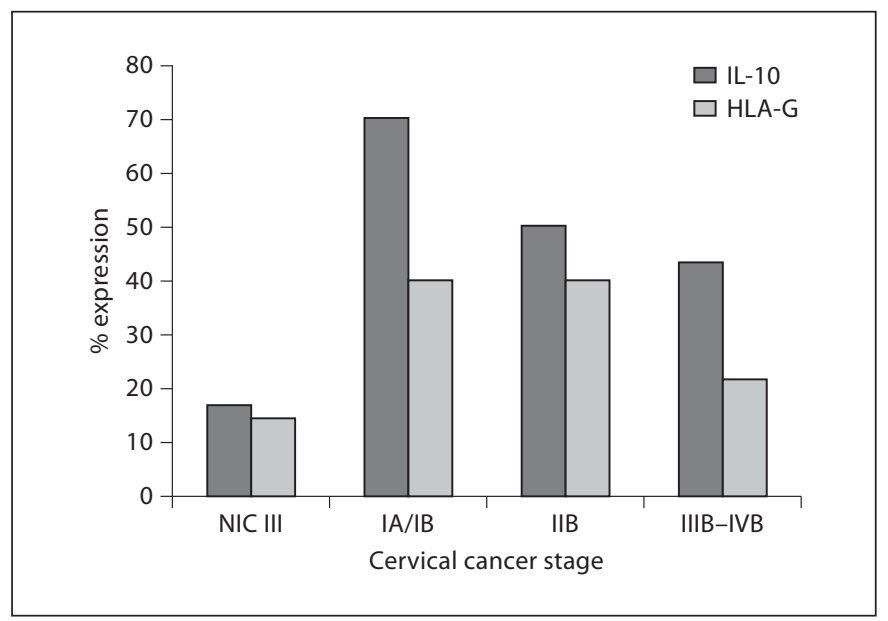

Fig. 4. HLA-G and IL-10 expression according to tumor stage of cervical cancer patients.

Table 4. Association of IL-10 expression with HLA class I expression and HLA-G expression

\begin{tabular}{|c|c|c|c|}
\hline & \multicolumn{3}{|c|}{ IL-10 expression } \\
\hline & negative, $\mathrm{n}$ & positive, $\mathrm{n}$ & $\mathrm{p}$ \\
\hline \multicolumn{4}{|l|}{ HLA class I expression } \\
\hline Negative $(n=38)$ & $25(80.6 \%)$ & $13(48.1 \%)$ & $0.028^{*}$ \\
\hline Weak expression $(\mathrm{n}=11)$ & $4(12.9 \%)$ & $7(25.9 \%)$ & \\
\hline Positive $(n=9)$ & $2(6.5 \%)$ & $7(25.9 \%)$ & \\
\hline Total $(n=58)$ & $31(100 \%)$ & $27(100)$ & \\
\hline \multicolumn{4}{|l|}{ HLA-G expression } \\
\hline Negative $(\mathrm{n}=42)$ & $29(69.0 \%)$ & $13(31.0 \%)$ & $0.000^{* *}$ \\
\hline Positive $(\mathrm{n}=16)$ & $2(12.5 \%)$ & $14(87.5 \%)$ & \\
\hline
\end{tabular}

$\mathrm{p}<0.05$ indicates significant differences. ${ }^{*}$ Pearson's $\chi^{2}$ test. ** Fisher's exact test.

grade and tumor stage [38]. Figure 4 shows HLA-G and IL-10 expression at different stages of cervical cancer. Although HLA-G expression was observed even in the early stage of cervical cancer, it did not show a significant association with the tumor stages.

\section{Analysis of HLA Class I, HLA-G and IL-10 on}

Survival

We analyzed the role of IL-10, HLA-G and HLA class I expression on overall survival. Although the patients that showed HLA-G and IL-10 expression had a shorter

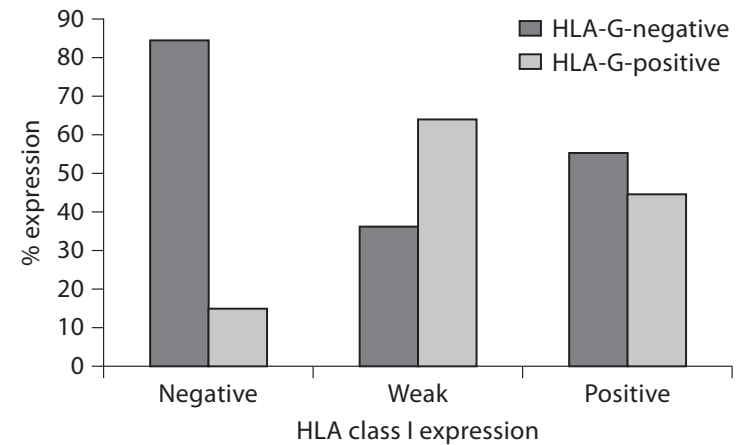

Fig. 5. Association of HLA-G expression and downregulation of HLA class I expression.

survival rate compared to those that did not have expression, this difference was not significant $(\mathrm{p}>0.5)$ (data not shown). Similarly, a non-significant association was observed between total loss or HLA class I downregulation and decreased overall survival.

\section{Association of HLA-G Expression and \\ Downregulation of HLA Class I Expression}

When the association between HLA-G and HLA class I expression was analyzed, we observed that among the patients who had a total loss of HLA class I expression, 15.4\% (6/39) expressed HLA-G. Among patients with HLA class I weak expression, 63.6\% (7/11) expressed HLA-G. The expression of HLA-G in HLA class I-positive cases was 44.4\% (4/9; Pearson's $\chi^{2}, \mathrm{p}=0.004$; fig. 5).

\section{Discussion}

Lymphoproliferative responses to specific HPV 16 E6 and E7 peptides appear to be associated with the clearance of HPV infection and with the regression of cervical lesions [39]. However, an increase of HPV-16 E7-specific T-helper type 1 response in peripheral blood of cervical cancer patients after radiotherapy is not enough for controlling tumor cell regression [40]. This observation underlines the importance of several mechanisms that could influence the anti-tumoral immune response. A shift from a Th1 to a Th2 cytokine profile with subse- 
quent IL-10 expression has been associated with impairment of tumor immune surveillance and progression of cervical cancer $[23,24]$. Furthermore, other cancer models indicate that IL-10 expression may contribute to impaired anti-tumor response through downregulating HLA class I and the selective HLA-G expression allowing tumor progression [30-32, 41]. In this study, we analyzed the relationship between $\mathrm{LOH}$ in the $6 \mathrm{p} 21$ and 15 q21 regions and HLA class I, HLA-G and IL-10 expression in biopsies of cervical cancer patients in order to comprehend how these alterations could contribute to evasion of immune surveillance control in cervical cancer.

Downregulation of HLA class I expression has been reported in many tumors of different origin and represents an important mechanism for tumors to escape from immune surveillance $[8,9]$. It eventually leads to accumulation of new tumor variants with low immunogenicity and high capability for metastatic progression $[7,42,43]$. In addition, it has been reported that loss of a $6 \mathrm{p}$ haplotype reduces the cell's heterozygous advantage to present a wide variety of tumor antigens in the context of HLA, for recognition by CTLs [44]. In cervical cancer, $\mathrm{LOH}$ very frequently occurs in the $6 \mathrm{p} 21.3$ region and represents an important common mechanism by which HLA genes and their products are abolished [45]. In this study, we first analyzed $\mathrm{LOH}$ in the $6 \mathrm{p} 21.3$ region in 63 cancer patients. As we had observed previously [35], the frequency of this HLA alteration varied from 24.1 to $62 \%$ depending on the STR marker. Patients with advanced stages of cervical cancer had greater frequencies of $\mathrm{LOH}$ than patients with CIN III. Nevertheless, the only significant difference was observed when we compared the CIN III group with invasive stage IV (data not shown). These results are in accordance with previous studies and extend the data reported by Vermeulen and coworkers [13,45] and Koopman et al. [14], who suggested that $\mathrm{LOH}$ in the $6 \mathrm{p} 21.3$ region occurs early and is frequently observed in cervical carcinogenesis. Moreover, a higher haplotype loss was observed in advanced stages of cervical cancer, supporting the concept that the $6 \mathrm{p} 21.3$ region, which carries HLA genes, is unstable during malignant transformation of cells, contributing to the mechanism of tumor escape and metastatic progression [46].

It has been observed that $\mathrm{LOH}$ in the $6 \mathrm{p} 21.3$ region is a frequent mechanism that leads to HLA class I abnormalities in other cancer models [47]. In this study, no correlation between $\mathrm{LOH}$ in the $6 \mathrm{p} 21.3$ region and HLA class I downregulation was found. Although a previous study reported that $\mathrm{LOH}$ in the $6 \mathrm{p} 21.3$ region is a frequent event occurring in tumors with downregulation of HLA class I expression compared to those that have a normal HLA class I expression [36], in our study a high percentage of tumors with normal HLA class I expression showed LOH and haplotype loss. It can be explained by the fact that $\mathrm{W} 6 / 32 \mathrm{mAb}$ used in this study recognizes surface-expressed HLA class I molecules associated with $\beta_{2} \mathrm{~m}$, but not allele-specific molecules of HLA class I, and there are still a number of important HLA allele specificities that cannot be defined in tissue because of the lack of an appropriate anti-HLA mAb $[34,37]$. On the other hand, some cases (5) with total loss of HLA class I expression did not have LOH. These results could also suggest that decrease of HLA class I expression may be due to mechanisms other than $\mathrm{LOH}$ in the $6 \mathrm{p} 21.3$ region.

In cervical cancer, it has been observed that the expression of anti-inflammatory cytokines such as IL-4, IL-10 and TGF- $\beta$ correlates with disease severity [48]. Moreover, the expression of anti-inflammatory cytokines in a cervical tumor microenvironment can lead to local immunosuppression, which is associated with deregulation of several molecules of the immune response [49] including downregulation of MHC class I expression [50-52]. In this study, loss or downregulation of HLA class I expression among patients who expressed IL-10 was found, supporting the concept that the alteration of HLA class I expression could be caused by the presence of IL-10 in the tumor environment [30]. Nevertheless, we found some cases without IL-10 expression that showed a downregulation of MHC class I expression. It has been reported that E6 and E7 oncoproteins of high-risk HPVs can disturb cell-cycle control and downregulate surface HLA class I antigens by decreasing components of the antigen presentation pathway [53]. In this study, most of the patients were HPV 16 infected, and it is possible that this event could contribute to the HLA downregulation in the tumor milieu.

HLA-G is a non-classical MHC molecule detected on extravillous cytotrophoblasts which plays an important role in immune tolerance by inhibiting the cytotoxic functions of T cells and NK cells [54], and in tumor cells its expression contributes to evasion of immune surveillance $[27,28,32,55]$. In the present study, cervical lesions of different stages were evaluated for HLA-G expression. Contrary to what was reported by Zhou et al. [56], who observed a strong and uniform HLA-G expression in normal epithelium, while only a small proportion of CINs and squamous cell carcinoma samples showed reduced expression of HLA-G, our results show upregulation of HLA-G from the early stages of cervical cancer 
which increased in a malignant lesion; however, a diminution was observed in the more advanced stage. These results correlate with the report by Yoon et al. [33], who found high HLA-G mRNA expression associated with early-stage cervical cancer and support a probable role for HLA-G in early carcinogenesis. In another study, Goncalves et al. [57] reported similar results: HLA-G5 isoform molecules were detected by immunohistochemistry in 25 cases of 74 cervical cancer biopsies (31.6\%), 17 (32.7\%) without metastasis and 8 (29.6\%) with metastasis. Moreover, a low expression of the HLA-G5 isoform was observed in the majority of HPV-related cases [58]. In this report the authors suggest that HPV may be involved in the modulation of HLA-G surface expression by using similar mechanisms to those observed in HLA class I downregulation by HPV oncoproteins; however, it is well-known that the HLA-G-immunosuppressive effect is mediated by upregulation of this molecule.

On the other hand, in another report, Dong et al. [59] found that in cervical cancer lesions, HLA-G is associated not only with disease progression but also with HPV infection. They observed a significantly higher HLA-G expression in CIN lesions infected with HPV 16/18 and cervical cancer than in HPV-negative patients. In our study, most of the patients were HPV 16 infected, and it is possible that this event could contribute to the HLA-G expression; however, the molecular mechanisms of HPV involved in the modulation of HLA-G expression are unknown. In addition, the mechanisms underlying the differential expression of HLA-G within a tumor type and among different tumors remain to be determined, but it may be influenced by tumor microenvironment as well as by the pathogenesis underlying the malignant transformation of the cells such as surrounding cytokine profile, epigenetic modifications and stress such as hypoxia $[22,60]$.

In human cancer, IL-10 expression appears to be one of the factors responsible for the upregulation of HLA-G $[32,41]$. In a previous report, Yoon et al. [33] reported that expression of both HLA-G and IL-10 in cervical cancer might play an important role in cervical cancer progression. However, in their study there was no significant correlation between HLA-G and IL-10 expression both at the levels of mRNA and protein. In this study, we found that IL-10 was expressed in $46.6 \%$ of cases while HLA-G was expressed in $27.6 \%$ of cases. Moreover, we also observed that most of the HLA-G-positive cases (14/16, 87.5\%) exhibited upregulation of the IL-10 cytokine $(\mathrm{p}=0.000)$. These results are in agreement with those reported by Urosevic and Dummer [61] in lung cancer where $77 \%$ of HLA-G-positive cases overexpressed IL-10 cytokine. Given the fact that the tumors themselves as well as tumor-infiltrating cells often produce IL-10 [62], and that IL-10-producing cells often localize in the vicinity of HLA-G-expressing cells, it is conceivable that IL-10 might be one of the factors responsible for HLA-G upregulation in cancer [61]. Thus, our results extend the previous findings reported by Urosevic et al. [32] suggesting that IL-10 might be one of the factors responsible for the upregulation of HLA-G and suggest, like other cancer models, that HLA-G could be another mechanism involved in immune escape in cervical cancer.

Finally, HLA-G expression has been proposed as a highly specific marker for malignant transformation [63] and can be useful in predicting the clinical outcome in some cancer patients [64-66]. However, in this study there were no significant differences in overall survival rate between patients with or without HLA-G expression. Moreover, HLA-G expression was not directly associated with the tumor stage. In accordance with other cancer models [67], it is possible that in cervical cancer, as suggested by Yoon et al. [33], HLA-G may be involved earlier in the course of malignant transformation, decreasing in the latter invasion stages, but may not be a prognostic factor [33]. Although our study is limited due to the small number of patients, further studies are necessary to resolve the clinical implications of HLA-G expression in cervical cancer.

Overall, our results suggest that in cervical cancer, the tumor microenvironment characterized by IL-10 secretion may induce an immunosuppressive environment by upregulating HLA-G expression and downregulating HLA class I expression, which can conduce to a low susceptibility to specific CTL-mediated lysis, and decreased NK susceptibility. These results could have important implications for identification of patients who could benefit from new therapeutic strategies, particularly in those selected for T-cell-based immunotherapies.

\section{Acknowledgments}

The authors sincerely thank all the institutions and individuals who participated in this study for their generosity. We thank the Gynecology Group of the Instituto Nacional de Cancerología, Bogotá, Colombia, especially Dr. Mónica Medina. This work was supported by Colciencias grant 21010413021 and INC/DNP 41030310-7. 


\section{References}

1 Frazer IH: The role of the immune system in anogenital human papillomavirus. Australas J Dermatol 1998;39(suppl 1):S5-S7.

2 Melbye M, Palefsky J, Gonzales J, Ryder LP, Nielsen H, Bergmann O, Pindborg J, Biggar RJ: Immune status as a determinant of human papillomavirus detection and its association with anal epithelial abnormalities. Int J Cancer 1990;46:203-206.

-3 Palefsky J: Human papillomavirus-associated malignancies in HIV-positive men and women. Curr Opin Oncol 1995;7:437-441.

4 Rezza G, Giuliani M, Serraino D, Branca M, Benedetto A, Garbuglia A, Ippolito G, Franceschi S: Risk factors for cervical presence of human papillomavirus DNA among women at risk for HIV infection. DIANAIDS Collaborative Study Group. Epidemiol Infect 1998;121:173-177.

5 Garcia-Lora A, Algarra I, Garrido F: MHC class I antigens, immune surveillance, and tumor immune escape. J Cell Physiol 2003; 195:346-355.

-6 Garrido F, Algarra I: MHC antigens and tumor escape from immune surveillance. Adv Cancer Res 2001;83:117-158.

-7 Garrido F, Cabrera T, Concha A, Glew S, Ruiz-Cabello F, Stern PL: Natural history of HLA expression during tumour development. Immunol Today 1993;14:491-499.

-8 Garrido F, Cabrera T, Lopez-Nevot MA, Ruiz-Cabello F: HLA class I antigens in human tumors. Adv Cancer Res 1995;67:155195.

-9 Garrido F, Ruiz-Cabello F, Cabrera T, PerezVillar JJ, Lopez-Botet M, Duggan-Keen M, Stern PL: Implications for immunosurveillance of altered HLA class I phenotypes in human tumours. Immunol Today 1997;18: 89-95.

10 Breitburd F, Ramoz N, Salmon J, Orth G: HLA control in the progression of human papillomavirus infections. Semin Cancer Biol 1996;7:359-371.

11 Chil A, Sikorski M, Bobek M, Jakiel G, Marcinkiewicz J: Alterations in the expression of selected MHC antigens in premalignant lesions and squamous carcinomas of the uterine cervix. Acta Obstet Gynecol Scand 2003; 82:1146-1152.

12 Hilders CG, Munoz IM, Nooyen Y, Fleuren GJ: Altered HLA expression by metastatic cervical carcinoma cells as a factor in impaired immune surveillance. Gynecol Oncol 1995;57:366-375.

13 Vermeulen CF, Jordanova ES, ZomerdijkNooijen YA, ter Haar NT, Peters AA, Fleuren GJ: Frequent HLA class I loss is an early event in cervical carcinogenesis. Hum Immunol 2005;66:1167-1173.

- 14 Koopman LA, Corver WE, van der Slik AR, Giphart MJ, Fleuren GJ: Multiple genetic alterations cause frequent and heterogeneous human histocompatibility leukocyte antigen class I loss in cervical cancer. J Exp Med 2000;191:961-976.
15 Mazurenko NN, Bliev AI, Bidzhieva BA, Peskov DI, Snigur NV, Savinova EB, Kiselev FL: Loss of heterozygosity at chromosome 6 as a marker of early genetic alterations in cervical intraepithelial neoplasias and microinvasive carcinomas (in Russian). Mol Biol (Mosk) 2006;40:436-447.

$\checkmark 16$ Carosella ED, Favier B, Rouas-Freiss N, Moreau P, LeMaoult J: Beyond the increasing complexity of the immunomodulatory HLA-G molecule. Blood 2008;111:48624870.

17 Le Gal FA, Riteau B, Sedlik C, Khalil-Daher I, Menier C, Dausset J, Guillet JG, Carosella ED, Rouas-Freiss N: HLA-G-mediated inhibition of antigen-specific cytotoxic T lymphocytes. Int Immunol 1999;11:1351-1356.

18 Marchal-Bras-Goncalves R, Rouas-Freiss N, Connan F, Choppin J, Dausset J, Carosella ED, Kirszenbaum M, Guillet J: A soluble HLA-G protein that inhibits natural killer cell-mediated cytotoxicity. Transplant Proc 2001;33:2355-2359.

19 Gros F, Cabillic F, Toutirais O, Maux AL, Sebti Y, Amiot L: Soluble HLA-G molecules impair natural killer/dendritic cell crosstalk via inhibition of dendritic cells. Eur J Immunol 2008;38:742-749.

20 Agaugue S, Carosella ED, Rouas-Freiss N: Role of HLA-G in tumor escape through expansion of myeloid-derived suppressor cells and cytokinic balance in favor of Th2 versus Th1/Th17. Blood 2011;117:7021-7031.

21 Zidi I, Ben AN: HLA-G as predisposing for metastasis. Med Hypotheses 2011;77:134139.

22 Yan WH: Human leukocyte antigen-G in cancer: are they clinically relevant? Cancer Lett 2011;311:123-130.

23 Bais AG, Beckmann I, Ewing PC, Eijkemans MJ, Meijer CJ, Snijders PJ, Helmerhorst TJ: Cytokine release in HR-HPV+ women without and with cervical dysplasia (CIN II and III) or carcinoma, compared with HR-HPVcontrols. Mediators Inflamm 2007;2007: 24147.

24 Clerici M, Merola M, Ferrario E, Trabattoni D, Villa ML, Stefanon B, Venzon DJ, Shearer GM, De Palo G, Clerici E: Cytokine production patterns in cervical intraepithelial neoplasia: association with human papillomavirus infection. J Natl Cancer Inst 1997;89: 245-250.

25 Kim J, Modlin RL, Moy RL, Dubinett SM, McHugh T, Nickoloff BJ, Uyemura K: IL-10 production in cutaneous basal and squamous cell carcinomas. A mechanism for evading the local T-cell immune response. J Immunol 1995; 155:2240-2247.

26 Pazmany L, Mandelboim O, Vales-Gomez M, Davis DM, Becker TC, Reyburn HT, Seebach JD, Hill JA, Strominger JL: Human leucocyte antigen- $G$ and its recognition by natural killer cells. J Reprod Immunol 1999;43: 127-137.
27 Paul P, Rouas-Freiss N, Khalil-Daher I, Moreau P, Riteau B, Le Gal FA, Avril MF, Dausset J, Guillet JG, Carosella ED: HLA-G expression in melanoma: a way for tumor cells to escape from immunosurveillance. Proc Natl Acad Sci USA 1998;95:4510-4515.

-28 Fukushima Y, Oshika Y, Nakamura M, Tokunaga T, Hatanaka H, Abe Y, Yamazaki H, Kijima H, Ueyama Y, Tamaoki N: Increased expression of human histocompatibility leukocyte antigen- $\mathrm{G}$ in colorectal cancer cells. Int J Mol Med 1998;2:349-351.

29 Riteau B, Menier C, Khalil-Daher I, Martinozzi S, Pla M, Dausset J, Carosella ED, Rouas-Freiss N: HLA-G1 co-expression boosts the HLA class I-mediated NK lysis inhibition. Int Immunol 2001;13:193-201.

30 Matsuda M, Salazar F, Petersson M, Masucci G, Hansson J, Pisa P, Zhang QJ, Masucci MG, Kiessling R: Interleukin-10 pretreatment protects target cells from tumor- and allospecific cytotoxic $\mathrm{T}$ cells and downregulates HLA class I expression. J Exp Med 1994;180: 2371-2376.

31 Sharma S, Stolina M, Lin Y, Gardner B, Miller PW, Kronenberg M, Dubinett SM: T cellderived IL-10 promotes lung cancer growth by suppressing both T-cell and APC function. J Immunol 1999;163:5020-5028.

- 32 Urosevic M, Kurrer MO, Kamarashev J, Mueller B, Weder W, Burg G, Stahel RA, Dummer R, Trojan A: Human leukocyte antigen-G up-regulation in lung cancer associates with high-grade histology, human leukocyte antigen class I loss and interleukin- 10 production. Am J Pathol 2001;159:817-824.

-33 Yoon BS, Kim YT, Kim JW, Kim SH, Kim JH, Kim SW: Expression of human leukocyte antigen- $G$ and its correlation with interleukin-10 expression in cervical carcinoma. Int J Gynaecol Obstet 2007;98:48-53.

34 Ramal LM, Feenstra M, van der Zwan AW, Collado A, Lopez-Nevot MA, Tilanus M, Garrido F: Criteria to define HLA haplotype loss in human solid tumors. Tissue Antigens 2000;55:443-448.

35 Rodríguez JA, Galeano L, Palacios DM, Serrano ML, Bravo MM, Combita AL: Frecuencias de las pérdidas de heterocigocidad en la región que codifica para HLA en biopsias de pacientes con cáncer de cuello uterino. Rev Colomb Cancerol 2009;13:191-204.

36 Feenstra M, Veltkamp M, van Kuik J, Wiertsema S, Slootweg P, van den TJ, de Weger R, Tilanus M: HLA class I expression and chromosomal deletions at $6 \mathrm{p}$ and $15 \mathrm{q}$ in head and neck squamous cell carcinomas. Tissue Antigens 1999;54:235-245.

- 37 Maleno I, Cabrera CM, Cabrera T, Paco L, Lopez-Nevot MA, Collado A, Ferron A, Garrido F: Distribution of HLA class I altered phenotypes in colorectal carcinomas: high frequency of HLA haplotype loss associated with loss of heterozygosity in chromosome region 6p21. Immunogenetics 2004;56:244253. 
38 Chen HX, Lin A, Shen CJ, Zhen R, Chen BG, Zhang X, Cao FL, Zhang JG, Yan WH: Upregulation of human leukocyte antigen-G expression and its clinical significance in ductal breast cancer. Hum Immunol 2010;71: 892-898.

- 39 Kadish AS, Ho GY, Burk RD, Wang Y, Romney SL, Ledwidge R, Angeletti RH: Lymphoproliferative responses to human papillomavirus (HPV) type 16 proteins E6 and E7: outcome of HPV infection and associated neoplasia. J Natl Cancer Inst 1997;89:12851293.

-40 Delgado FG, Martinez E, Cespedes MA, Bravo MM, Navas MC, Combita Rojas AL: Increase of human papillomavirus-16 E7-specific T-helper type 1 response in peripheral blood of cervical cancer patients after radiotherapy. Immunology 2009;126:523-534.

-41 Urosevic M, Willers J, Mueller B, Kempf W, Burg G, Dummer R: HLA-G protein up-regulation in primary cutaneous lymphomas is associated with interleukin-10 expression in large cell T-cell lymphomas and indolent Bcell lymphomas. Blood 2002;99:609-617.

-42 Aptsiauri N, Cabrera T, Mendez R, GarciaLora A, Ruiz-Cabello F, Garrido F: Role of altered expression of HLA class I molecules in cancer progression. Adv Exp Med Biol 2007;601:123-131.

43 Chang CC, Ferrone S: Immune selective pressure and HLA class I antigen defects in malignant lesions. Cancer Immunol Immunother 2007;56:227-236.

-44 Boon T, van der Bruggen P: Human tumor antigens recognized by $\mathrm{T}$ lymphocytes. J Exp Med 1996;183:725-729.

45 Vermeulen CF, Jordanova ES, ter Haar NT, Kolkman-Uljee SM, de Miranda NF, Ferrone S, Peters AA, Fleuren GJ: Expression and genetic analysis of transporter associated with antigen processing in cervical carcinoma. Gynecol Oncol 2007;105:593-599.

- 46 Cabrera T, Lara E, Romero JM, Maleno I, Real LM, Ruiz-Cabello F, Valero P, Camacho FM, Garrido F: HLA class I expression in metastatic melanoma correlates with tumor development during autologous vaccination. Cancer Immunol Immunother 2007;56:709717.

47 Yang Y, Zhang J, Miao F, Wei J, Shen C, Shen Y, Xie W: Loss of heterozygosity at 6p21 underlying [corrected] HLA class I downregulation in Chinese primary esophageal squamous cell carcinomas. Tissue Antigens 2008; 72:105-114.
48 de Gruijl TD, Bontkes HJ, van den Muysenberg AJ, van Oostveen JW, Stukart MJ, Verheijen RH, van der Vange N, Snijders PJ, Meijer CJ, Walboomers JM, Scheper RJ: Differences in cytokine mRNA profiles between premalignant and malignant lesions of the uterine cervix. Eur J Cancer 1999;35:490497.

49 Sheu BC, Lin RH, Lien HC, Ho HN, Hsu SM, Huang SC: Predominant Th2/Tc2 polarity of tumor-infiltrating lymphocytes in human cervical cancer. J Immunol 2001;167:29722978.

50 Bermudez-Morales VH, Gutierrez LX, Cocer-Gonzalez JM, Burguete A, Madrid-Marina V: Correlation between IL-10 gene expression and HPV infection in cervical cancer: a mechanism for immune response escape. Cancer Invest 2008;26:1037-1043.

51 Ritz U, Momburg F, Pilch H, Huber C, Maeurer MJ, Seliger B: Deficient expression of components of the MHC class I antigen processing machinery in human cervical carcinoma. Int J Oncol 2001;19:1211-1220.

- 52 Sheu BC, Chiou SH, Chang WC, Chow SN, Lin HH, Chen RJ, Huang SC, Ho HN, Hsu SM: Integration of high-risk human papillomavirus DNA correlates with HLA genotype aberration and reduced HLA class I molecule expression in human cervical carcinoma. Clin Immunol 2005;115:295-301.

53 Li W, Deng XM, Wang CX, Zhang X, Zheng GX, Zhang J, Feng JB: Down-regulation of HLA class I antigen in human papillomavirus type $16 \mathrm{E} 7$ expressing $\mathrm{HaCaT}$ cells: correlate with TAP-1 expression. Int J Gynecol Cancer 2010;20:227-232.

54 Moreau P, Drian-Cabestre F, Menier C, Guiard V, Gourand L, Dausset J, Carosella ED, Paul P: IL-10 selectively induces HLA-G expression in human trophoblasts and monocytes. Int Immunol 1999;11:803-811.

55 Bukur J, Rebmann V, Grosse-Wilde H, Luboldt H, Ruebben H, Drexler I, Sutter G, Huber C, Seliger B: Functional role of human leukocyte antigen-G up-regulation in renal cell carcinoma. Cancer Res 2003;63:41074111.

56 Zhou JH, Ye F, Chen HZ, Zhou CY, Lu WG, Xie X: Altered expression of cellular membrane molecules of HLA-DR, HLA-G and CD99 in cervical intraepithelial neoplasias and invasive squamous cell carcinoma. Life Sci 2006;78:2643-2649.
57 Goncalves MA, Le DM, Simoes RT, Rabreau M, Soares EG, Donadi EA, Carosella ED: Classical and non-classical HLA molecules and p16(INK4a) expression in precursors lesions and invasive cervical cancer. Eur J Obstet Gynecol Reprod Biol 2008;141:70-74.

58 Guimaraes MC, Soares CP, Donadi EA, Derchain SF, Andrade LA, Silva TG, Hassumi MK, Simoes RT, Miranda FA, Lira RC, Crispim J, Soares EG: Low expression of human histocompatibility soluble leukocyte antigen-G (HLA-G5) in invasive cervical cancer with and without metastasis, associated with papilloma virus (HPV). J Histochem Cytochem 2010;58:405-411.

-59 Dong DD, Yang H, Li K, Xu G, Song LH, Fan XL, Jiang XL, Yie SM: Human leukocyte antigen- $G$ expression in cervical lesions: association with cancer progression, HPV 16/18 infection, and host immune response. Reprod Sci 2010;17:718-723.

60 Moreau P, Flajollet S, Carosella ED: Nonclassical transcriptional regulation of HLAG: an update. J Cell Mol Med 2009;13:29732989.

61 Urosevic M, Dummer R: HLA-G and IL-10 expression in human cancer-different stories with the same message. Semin Cancer Biol 2003; 13:337-342.

62 Mocellin S, Wang E, Marincola FM: Cytokines and immune response in the tumor microenvironment. J Immunother 2001;24: 392-407.

-63 Barrier BF, Kendall BS, Sharpe-Timms KL, Kost ER: Characterization of human leukocyte antigen-G expression in endometrial adenocarcinoma. Gynecol Oncol 2006;103: 25-30.

64 Wang Y, Ye Z, Meng XQ, Zheng SS: Expression of HLA-G in patients with hepatocellular carcinoma. Hepatobiliary Pancreat Dis Int 2011;10:158-163.

65 Ye SR, Yang H, Li K, Dong DD, Lin XM, Yie SM: Human leukocyte antigen-G expression: as a significant prognostic indicator for patients with colorectal cancer. Mod Pathol 2007;20:375-383.

66 Yie SM, Yang H, Ye SR, Li K, Dong DD, Lin $\mathrm{XM}$ : Expression of human leukocyte antigen-G (HLA-G) correlates with poor prognosis in gastric carcinoma. Ann Surg Oncol 2007; 14:2721-2729.

67 Ishigami S, Natsugoe S, Miyazono F, Nakajo A, Tokuda K, Matsumoto M, Okumura H, Douchi T, Hokita S, Aikou T: HLA-G expression in gastric cancer. Anticancer Res 2006; 26:2467-2472. 